\title{
Influence of Mechanical Stress on Magnetization Processes in Rayleigh Area of Amorphous FINEMET-Type Alloy
}

\author{
J. KOVÁC̆ $\check{C}^{a *}$ AND L. NOVÁK ${ }^{b}$ \\ ${ }^{a}$ Institute of Experimental Physics SAS, Watsonova 47, 04001 Košice, Slovakia \\ ${ }^{b}$ Department of Physics, Technical University of Košice, Park Komenského 2, 04200 Košice, Slovakia \\ We have investigated the effect of mechanical stress on magnetization processes in FINEMET-type alloy \\ in region of the weak magnetic fields (Rayleigh area). The rapidly quenched FINEMET ribbons in the original \\ amorphous state have been chosen for the study. We have found significant differences in behavior of studied \\ materials at different values of applied mechanical stresses. These differences are ascribed to the changes in total \\ magnetic anisotropy and consequently also to the modifications in domain wall thickness.
}

DOI: 10.12693/APhysPolA.137.1003

PACS/topics: amorphous alloys, magnetization processes, mechanical stress

\section{Introduction}

The magnetization of ferromagnetic material can be described by four processes: the reversible and irreversible shift of domain walls, the rotation of the magnetic polarization vector, and paraprocess. The motion or shift of domain walls is characteristic process for the area of weak magnetic fields, whic is the so called Rayleigh region. In this area, the dependence of the magnetic polarization $J$ on magnetic field strength $H$ in the first approximation can be described by the following expression [1]

$$
J=\mu_{0} \kappa_{p} H+\alpha H^{2},
$$

where $\mu_{0}$ is the permeability of vacuum, $\kappa_{p}$ denotes initial volume magnetic susceptibility, and $\alpha$ is the Rayleigh constant. All these parameters are dependent on the type of the ferromagnetic material and its state.

The such defined dependence of magnetic polarization $J$ takes into account reversible (the first term) as well as irreversible (the second term) types of processes. The present paper focuses on the study of the magnetization processes in Rayleigh region influenced by different machanical stress conditions in the amorphous material.

For most ferromagnetic materials, mechanical stress is an important factor affecting their magnetic properties $[2,3]$. This is so significant that it can be considered as one of the fundamental factors influencing the magnetic properties of ferromagnetics, together with the strength of magnetic field and temperature. We decided to investigate the impact of mechanical stress (in the range of $\sigma=0-80 \mathrm{MPa}$ ) to magnetization processes in Rayleigh region of the FINEMET sample.

\footnotetext{
* corresponding author; e-mail: jkovac@saske.sk
}

\section{Methodology of the measurement}

The magnetic measurements have been performed on the FINEMET-type ribbons with chemical composition of $\left(\mathrm{Fe}_{73,5} \mathrm{Cu}_{1} \mathrm{Nb}_{3} \mathrm{Si}_{13.5} \mathrm{~B}_{9}\right)$ in as-cast state (amorphous). The dimension of the samples have been $10 \times 100 \times 0.022 \mathrm{~mm}^{3}$. The relevant magnetic parameters have been determined by using the magnetometer measured stray fields of the sample $[4,5]$.

The following magnetic parameters have been determined from the large hysteresis loop - the saturated magnetic polarization $J_{s}$, measured in the magnetic field of $15 \mathrm{kA} / \mathrm{m}$, the coercivity $H_{c}$, and the total magnetic anisotropy $K_{\text {eff }}$, determined from the area above the curve of the initial magnetic polarization. The values of $J_{s}$ have been constant, $J_{s}=1.191 \mathrm{~T}$ regardless of mechanical stress loading. The typical behaviour of $K_{\text {eff }}$ and $H_{c}$ for different value of mechanical stress $\sigma$ are presented in Fig. 1. The reversibility of the investigated phenomenons under the mechanical stress have been verified by measuring of the magnetic quantities with and without the applied mechanical stress.

At the same time the initial part of virgin curves of analyzed samples have been measured in detail. Using the Rayleigh parabola fit (1), the intrinsic parameters $\kappa_{p}$ and $\alpha$ have been determined. Their values are listed in Table I.

\section{Results and discussion}

The initial part of virgin curves of analyzed samples together with Rayleigh parabola fits are shown in Fig. 2. The size of the area in which the Rayleigh parabola coincides with the initial virgin curve area, ergo Rayleigh region, is in range $0-10 \mathrm{~A} / \mathrm{m}$ for all analyzed values of mechanical stress. Surprisingly, we have detected that the size of Rayleigh region is almost independent on the applied mechanical stress $\sigma$. However, the magnitude of coercivity $H_{c}$ is very sensitive to its value (see Fig. 1) 


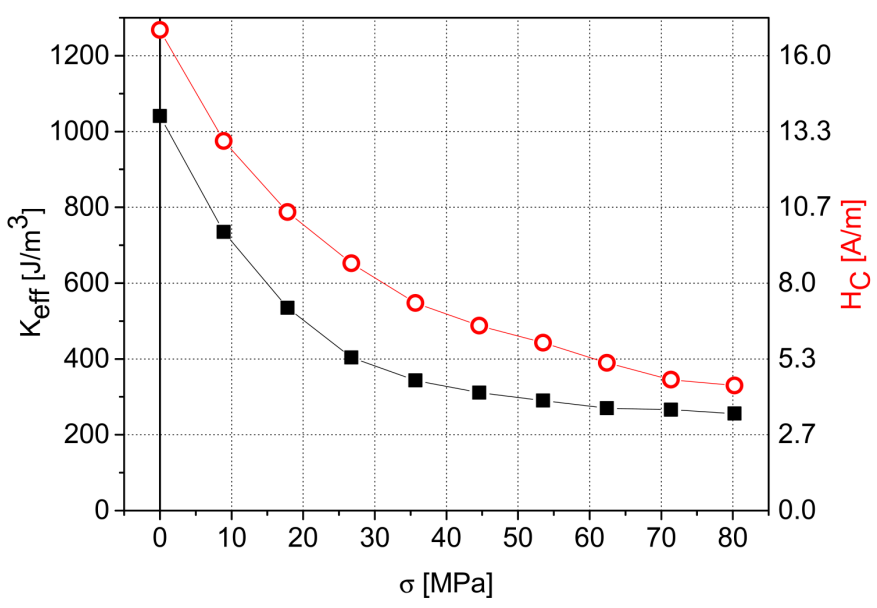

Fig. 1. The dependence of anisotropy $K_{\text {eff }}$ and coercivity $H_{c}$ as a function of external mechanical stress.

\section{TABLE I}

The parameters calculated from the measured data: the total magnetic anisotropy $K_{\text {eff }}$, initial volumetric magnetic susceptibility $\kappa_{p}$, Rayleigh constant $\alpha$, the relative size of the sample magnetized within Rayleigh region, i.e., the ratio of the maximum magnetic polarization achieved in Rayleigh region and saturated magnetic polarization of sample $J_{\text {Rayl }} / J_{s}$, and a thickness of $180^{\circ}$ domain walls $\delta_{w}$.

\begin{tabular}{c|c|c|c|c|c}
\hline $\begin{array}{c}\sigma \\
{[\mathrm{MPa}]}\end{array}$ & $\begin{array}{c}K_{\text {eff }} \\
{\left[\mathrm{J} / \mathrm{m}^{3}\right]}\end{array}$ & $\kappa_{p} \times 10^{-3}$ & $\begin{array}{c}\alpha \times 10^{5} \\
{\left[\mathrm{~Wb} / \mathrm{A}^{2}\right]}\end{array}$ & $\begin{array}{c}J_{\text {Rayl }} / J_{s} \\
{[\%]}\end{array}$ & $\begin{array}{c}\delta_{w} \\
{[\mathrm{~nm}]}\end{array}$ \\
\hline 0.00 & 1041 & 1.7 & 18 & 3.4 & 168 \\
8.92 & 735 & 2.0 & 33 & 5.0 & 200 \\
17.84 & 535 & 2.0 & 45 & 6.2 & 234 \\
26.75 & 404 & 2.5 & 65 & 8.8 & 270 \\
35.67 & 343 & 2.2 & 80 & 9.2 & 293 \\
44.59 & 311 & 2.0 & 93 & 10.1 & 308 \\
53.51 & 290 & 2.0 & 100 & 10.1 & 318 \\
62.43 & 270 & 2.0 & 101 & 10.5 & 330 \\
71.35 & 266 & 2.0 & 120 & 10.5 & 333 \\
80.26 & 256 & 2.0 & 105 & 10.9 & 339
\end{tabular}

The difference in size of the sample portion magnetized at different loads within the Rayleigh region is significant. This is the portion of the sample that has been magnetized at the given loading by the shift of the domain walls. If we compare the magnetic polarization achieved within Rayleigh region (value of magnetic polarization at magnetic field of $10 \mathrm{~A} / \mathrm{m}$ ) with saturated magnetic polarization (at field of $15 \mathrm{kA} / \mathrm{m}$ ), we can see that in the unloaded sample $3.4 \%$ of the total sample volume is magnetized. This ratio gradually increases to more than three times the original value when mechanical stress is applied on the sample (10.9\%), see Table I. It follows that the mobility of domain walls in Rayleigh region is significantly increased under mechanical stress. Commonly, such changes can be caused by decrease in the number of structural defects, which in our case are clusters or their

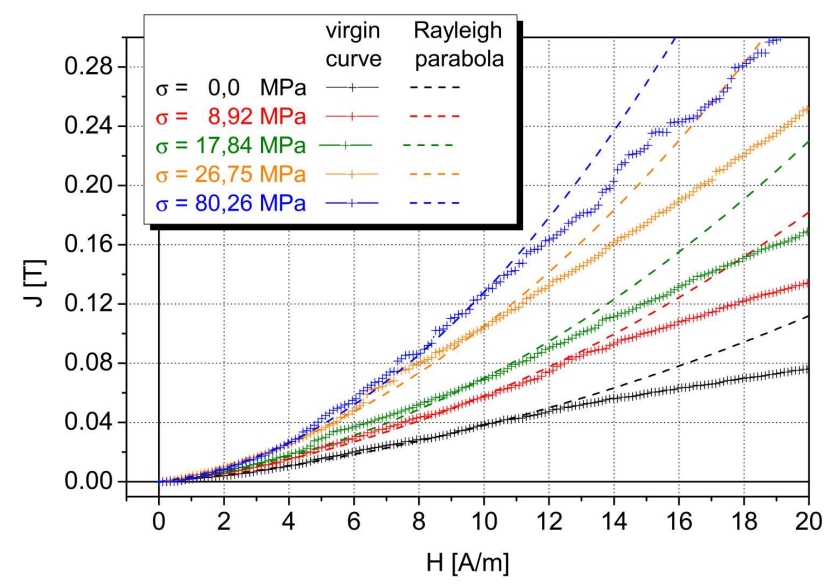

Fig. 2. The initial virgin curve area of magnetic polarization and the Rayleigh parabolas for FINEMET samples with different values of external mechanical stress.

reduction. However, such changes caused by mechanical loading are unrealistic. One possible explanation of such changes can be the change of domain wall thickness.

To estimate the thickness of $180^{\circ}$ domain walls, we can use a relationship $[2,6]$ :

$$
\delta_{w}=\pi \sqrt{\frac{A}{a K_{\mathrm{eff}}}} .
$$

Here, $A$ is the so called exchange integral defined as $A \doteq 0.15 k_{\mathrm{B}} T_{\mathrm{C}}$, where $k_{\mathrm{B}}$ is the Boltzmann constant $\left(k_{\mathrm{B}}=1.38 \times 10^{-23} \mathrm{~J} / \mathrm{K}\right)$, and $T_{\mathrm{C}}$ is the Curie temperature of the sample. The $T_{\mathrm{C}}$ value for FINEMET alloy is $T_{\mathrm{C}}=620 \mathrm{~K}[4,7]$. The lattice constant of the crystalline material $a$ denotes the distance between the atoms of amorphous alloy. For the FeSi alloy with a low Si content its value is $a=2.86 \times 10^{-10} \mathrm{~m}$. The above mentioned values of $T_{\mathrm{C}}$ and $a$ have been used for all further analyses.

FINEMET is a material with a relatively high anisotropy and coercivity. This is due to the technology of preparation which introduces a high degree of internal mechanical stresses into material. It causes a large magnetoelastic anisotropy in different directions. The distortions of structure caused by stress represent the barriers to the movement of domain walls. Due to the composition of the sample (containing up to 5 components), it may be also assumed that agglomeration, i.e., clustering of the atoms also occurs, which leads to a reduction in the mobility of the domain walls. On the contrary, the low mobility of the domain walls contributes to their thickness which at zero loading sample is comparable with dimensions of barriers.

For amorphous materials with non-zero magnetostriction in which crystallographic anisotropy is absent, the magnetoelastic anisotropy is determining. This anisotropy is a significantly predominant component of the effective anisotropy constant $\left(K_{\text {eff }}\right)$. Applying mechanical stress to the amorphous ribbon in the direction of the 
external field leads to the significant preference of the direction of magnetization, what is indicated by decrease in the effective anisotropy constant $K_{\text {eff }}$ value to one quarter after mechanical stress of $80 \mathrm{MPa}$ is applied. As a result, the domain wall thickness $\delta_{w}$, calculated by (2), is doubled. If we suppose that the dimensions of barriers in the movement direction of domain walls are not changed, they, in comparison to the wall thickness, seem to be diminishing. The bounding energy of the domain walls to these barriers decreases. This causes increase in the domains mobility $[5,8]$, what leads to three times smaller value of the coercivity during the loading process (see Fig. 1).

In addition, for the unloaded sample $(\sigma=0)$, the portion of the magnetized volume in the Rayleigh region $\left(J_{\text {Rayl }} / J_{s}\right)$ is small, resulting in small mobility of the domain walls. When the sample is loaded, the size of the sample volume magnetized in the Rayleigh region increases significantly depending on the applied stress. For example, when the stress of $\sigma=80 \mathrm{MPa}$ is applied to the sample, the sample volume magnetized in Rayleigh region is three times greater than at zero load (Table I). This is also proof of the increased domain walls mobility.

There are also other magnetic parameters that is related to the composition and structure. Mechanical stress does not affect them and they can be considered as material constants. Further, changes in initial volume magnetic susceptibility $\kappa_{p}$ are small and unsystematic during the process of loading, and therefore we can consider them as uncertainties in measurements. Even the initial volumetric magnetic susceptibility is not influenced by mechanical stress.

Surprisingly, the size of the Rayleigh region is also constant during the loading process $(0-10 \mathrm{~A} / \mathrm{m})$. We assume that the mechanical stress causes more volume of the sample to be magnetized within the Rayleigh region by reversing the movement of the domain walls than in the case without the load. However, the magnetic field that triggers the irreversible movement of the domain walls, regardless of the size of the mechanical stress loading, is the field $H_{i}=10 \mathrm{~A} / \mathrm{m}$.

\section{Conclusions}

There is no crystallographic anisotropy in the amorphous materials, and therefore, in the case of non-zero magnetostriction, the magnetoelastic anisotropy is dominant. Applying mechanical stress to the amorphous ribbon in the direction of the magnetic field leads to a significant preference of the direction of magnetization, which results in decrease in the effective anisotropy constant $K_{\text {eff }}$ value to one quarter for loading of stress of $80 \mathrm{MPa}$ in comparison with unloaded sample. As a result, the thickness of domain walls is doubled. By preserving the size of barriers to the movement of domain walls, this thickening of walls leads to a significant increase in their mobility. As a consequence of the significant increase in domain wall mobility, there is a threefold larger portion of the sample that is magnetized by the reversible shift of the domain walls within the RR.

\section{Acknowledgments}

This work has been supported by the VEGA Grants No. 1/0388/18 and 1/0053/19 from the Slovak Grant Agency of the Ministry of Education, Science, Research and Sport of the Slovak Republic.

\section{References}

[1] Lord R.S. Rayleigh Sec. Philos. Mag. 23, 225 (1887).

[2] S. Chikazumi, Phys. Ferromagn., Oxford University Press, 1997.

[3] M. Kachniarz, R. Szewczyk, J. Electr. Eng. 66, 82 (2015).

[4] J. Kováč, L. Novák, L. Hubač, J. Electr. Eng. 66, $142(2015)$.

[5] J. Kováč, L. Novák, J. Kecer, J. Alloys Compd. 735, 1591e1595 (2018).

[6] J.M.D. Coey, Magnetism and Magnetic Materials, Oxford University Press, Oxford, 2009.

[7] L. Novák, J. Kováč, L. Hubač, Acta Phys. Pol. A 131, 753 (2017).

[8] J. Kováč, L. Novák, Acta Elektrotech. Inform. 13, 61 (2013). 